\title{
On Formalizing Opportunism Based on Situation Calculus
}

\author{
Jieting Luo ${ }^{(凶)}$, John-Jules Meyer, and Frank Dignum \\ Utrecht University, PO Box 80089, 3508 TB Utrecht, The Netherlands \\ $\{$ J.Luo, J.J.C.Meyer, F.P.M.Dignum\}@uu.nl
}

\begin{abstract}
In social interactions, it is common for one party to possess more or better knowledge about a specific transaction than others. In this situation, parties who are more knowledgeable might perform opportunistic behavior to others, which is against others' interest thus leading to relationship deterioration. In this study, we propose formal models of opportunism, which consist of the properties knowledge asymmetry, value opposition and intention, based on situation calculus in different context settings. We illustrate our formalization through a simple example. Further study on its emergence and constraint mechanism can be carried out based on the formal models.
\end{abstract}

Keywords: Opportunism $\cdot$ Value $\cdot$ Situation calculus $\cdot$ Formalization

\section{Introduction}

Consider a common social interaction. A seller is trying to sell a cup to a buyer and it is known by the seller beforehand that the cup is actually broken (e.g. there is a crack at the bottom of the cup). The buyer buys the cup for its good appearance, but of course gets disappointed when he fills it with water. In this example, the seller earns money from the buyer by exploiting the opportunity of knowledge asymmetry about the cup, while the buyer just focuses on the appearance of the cup rather than being leaky or not. Such a social behavior intentionally performed by the seller is first proposed by economist Williamson as opportunism [1]. Opportunistic behavior commonly exists in business transactions and other types of social interactions in various forms such as deceit, lying and betraying.

Viewing individuals as agents, we may have similar problems in multi-agent system research. Interacting agents were modeled to behave in a human-like way with characteristics of autonomy, local views and decentralization [2]. When such agents possess different quantity or quality of relevant information and try to maximize their benefits, they may probably perform opportunistic behavior to others, which is against others' benefits or the norms of the system. For example, in a system with the norm of equity, an agent may hide important information to his or her peers for increasing his own payoff. The agent's behavior has negative results for other agents involved in the relationship and strongly affects the cooperative relationship once it is unveiled.

(C) Springer International Publishing Switzerland 2015

F. Koch et al. (Eds.): CARE-MFSC 2015, CCIS 541, pp. 147-166, 2015.

DOI: $10.1007 / 978-3-319-24804-2 \_10$ 
In order to explore this problem, we need to have a formal model of opportunism which can be applied in any context and serve as a basic framework for future research. Logic-based formalisms are one of the alternatives for its capacity of describing and reasoning. Through the specification by logic, we can understand more clearly the elements in the definition and how they constitute this social behavior. More importantly, we can derive interesting properties that are useful for our future research. Thus, we are motivated to propose a formal specification of opportunism by mathematical logic based on our definition. We believe that such a research perspective can ease the debates about opportunism in social science. Moreover, future work on its emergence and constraint mechanism can be conducted based on our formal model, rendering our study relevant for MAS research.

In this paper, we first have a clear definition of opportunism extended from Williamson's, highlighting the key elements we need to model. Aiming at the investigation about the different judgement on opportunistic behavior, we integrate the notion of value to represent agents' preference on situations. We then formalize opportunism using the situation calculus $[6,7]$ as our technical framework based on our extended definition. We first consider opportunistic behavior as a single action between two agents, and then extend it to multiple actions and incorporate social context in the model. Our formal models of opportunism consist of the property knowledge asymmetry, value opposition and intention, and represent how they relate to each other. We illustrate how to use our formal models through a simple example selling a broken cup.

\section{Defining Opportunism with Value}

In this section, we extend Williamson's definition of opportunism and suggest a more explicit one as a prelude and basis to proposing a formal model in the next section.

\subsection{Definition of Opportunism}

The classical definition of opportunism is offered by Williamson [1] as "selfinterest seeking with guile". While this definition has been used in a large amount of research, it only mentions two attributes, self-interest and guile, explicitly, leaving other attributes for researchers to interpret from different perspectives. For example, Das defined partner opportunism as "behavior by a partner firm that is motivated to pursue its self-interest with deceit to achieve gains at the expense of the other alliance members" [3]. Even though it is elaborated enough, it has the suggestion that opportunistic individuals are meant to harm others, which cannot be derived from Williamson's definition. In this study, based on the definition of Williamson, we compare opportunistic scenarios with nonopportunistic ones, and redefine this social behavior in a more explicit way. 
Opportunism is a behavior that is motivated by self-interest and takes advantage of relevant knowledge asymmetry ${ }^{1}$ to achieve gains, regardless of the principles.

First of all, there has been reached consensus that opportunistic behavior is performed with self-interest motivation [3]. We admit that self-interested pursuit is the natural property of human beings, but opportunism is more than that: individuals with opportunistic behavior do not care about the negative effects on others.

Secondly, relevant knowledge asymmetry provides the chance to individuals to be opportunistic. Opportunistic individuals may break the contracts or the relational norms using the relevant knowledge that others do not have. It is important for opportunistic individuals to use cheating, deceit or infidelity for hiding their self-interest motive. Therefore, individuals with more relevant knowledge will have more potential for opportunistic behavior.

Thirdly, principles are ignored by opportunistic individuals. The reason to use "ignore" here is to distinguish opportunism from accidentally bringing harm to others. Opportunistic behavior is performed on purpose without any compensation to the victims. Principles can be the value of others, or the contract rules or the relational norms that are used for balancing various interests and already agreed to by a majority of the individuals.

Fourthly, even though we did not explicitly declare the result of performing opportunistic behavior in our extended definition, such a social behavior must result in gains at the expense of others. Any self-interested behavior that does not affect other individuals should not be considered as opportunism.

From the above elaboration, we have something important to keep in mind: it is not the intention of opportunistic individuals to harm others even though opportunism is deliberate with self-interest motives. The ignored principles are a specific kind of knowledge about the interest of others that cannot be considered as an intention to be opportunistic. This is one of the properties that we are going to show through our formal model of opportunism.

\subsection{Integrating with Value}

Based on the informal definition of opportunism, the example about hiding information that we encountered in the introduction is opportunistic behavior, since it is against others' benefits or the norms of the system. However, if hiding is not forbidden by the norm, the agent could not be said to have done anything wrong. Or if other agents agree with that agents having more important information deserve more payoff, it may not be against other agents' interest. We can see that both the system's norms and the agents' perspectives can influence the judgement of opportunism, and they are the representation of value systems

\footnotetext{
${ }^{1}$ Even though many papers in social science use information asymmetry to represent the situation where one party in a transaction knows more compared to another, we would rather revise it as knowledge asymmetry in this paper for the purpose of being consistent with our technical framework of situation calculus and its extensions.
} 
at the collective level and individual level respectively, which may be different among systems and agents.

Value is something that we think is important, and various types of values together with their orderings form a value system. By integrating the notion of value into our model, the result of performing opportunistic behavior is represented as the promotion of opportunistic individuals' value and the demotion of others' value. Furthermore, even though a value system is relatively stable within individuals, it may differ across different individuals and societies. For societies, each has its own value system as part of the social context and it serves as the basis for any judgment within the society. In this sense, some behaviors which are regarded as opportunistic in one society may not be considered as opportunistic in another society, if the two societies do not share the same value system. A similar idea, although more focusing on opportunistic propensity, can be found in [5]. Given the value system of the society, opportunistic behavior promotes the self-interest which is in opposition with others' value.

\section{Technical Framework: Situation Calculus}

Situation calculus provides a formal language for representing and reasoning about dynamical domains based on first-order logic. There are three types of sorts: actions that can be performed by agents, situations representing a history of action occurrences and objects for everything else. Situation $S_{0}$ represents the initial situation that no action can result in. The special predicate $d o(a, s)$ denotes the unique situation that results from the performing of action $a$ in situation $s$. The properties of situations are specified through relational and functional fluents taking a situation term as their last argument, which means their truth value may vary from situation to situation. The effects of actions on fluents are defined by successor state axioms. Also propositions $P$ can be used instead of fluents, that is, their truth values are not dependent on the situation but consistent throughout all the situations.

With situation calculus, we can reason about how the world changes as the result of the available actions. A Basic Action Theory from Reiter [7] is defined as

$$
D=\Sigma \cup D_{a p} \cup D_{s s} \cup D_{\text {so }} \cup D_{\text {una }}
$$

$\Sigma$ : the set of foundational axioms,

$-d o\left(a_{1}, s_{1}\right)=d o\left(a_{2}, s_{2}\right) \rightarrow a_{1}=a_{2} \wedge s_{1}=s_{2}$;

- $(\forall Q) Q\left(S_{0}\right) \wedge(\forall s, a)[Q(s) \rightarrow Q(d o(a, s))] \rightarrow(\forall s) Q(s)$;

$-s \sqsubset d o\left(a, s^{\prime}\right) \equiv s \sqsubseteq s^{\prime}$;

$-\neg s \sqsubset S_{0}$;

$D_{a p}$ : the set of actions preconditions,

$$
\operatorname{Poss}(a(x), s) \equiv \pi_{a}(x, s)
$$


$D_{s s}$ : the set of successor state axioms,

$$
F(d o(a, s)) \equiv \gamma_{F}^{+}(a, s) \vee\left(F(s) \wedge \neg \gamma_{F}^{-}(a, s)\right)
$$

Here $\gamma_{F}^{+}(a, s)$ and $\gamma_{F}^{-}(a, s)$ are two formulas expressing the conditions for the fluent $F$ becoming true and false, respectively; $D_{s o}$ : the sentences uniform in $S_{0}$ describing the initial situation; $D_{\text {una }}$ : the unique name axioms for actions.

This is a brief overview of situation calculus, which is the technical preliminary of our formalization. However, this language can only provide information about the history of a situation and there is no way to represent the future of a situation. For example, propositions like "I sell the cup now" cannot be represented by situation calculus. Since this representation is of great importance to our formalization, we extend the situation to one-step further in the future. An extended situation is a pair $\left(s, s^{\prime}\right)$ such that $s$ is a situation and $s^{\prime}$ is the next situation of $s$, and occur is a relation between actions and situations. Here is the semantic of occur:

- $\left(s, s^{\prime}\right) \vDash \operatorname{occur}(a, s)$ iff $s^{\prime}=d o(a, s)$. That is, $\operatorname{occur}(a, s)$ holds if action $a$ occurs in situation $s$.

After John McCarthy's introduction of this theory, people made extensions capable of representing knowledge, belief, intention and obligation in order to better reason about actions and their effects on the world [8-10]. We will introduce and adopt those extensions in the following sections as appropriate. Since in situation calculus the last argument is always a situation, we will follow this convention in this paper for any definition of fluents and predicates.

\section{Formalizing Opportunism}

\subsection{Knowledge Asymmetry}

We adopt the approach of Scherl to formalizing knowledge, which is to add an agents' possible-world model of knowledge to situation calculus [9]. To treat knowledge as a fluent, we have a binary relation $K\left(s^{\prime}, s\right)$, reading as situation $s^{\prime}$ is epistemically accessible from situation $s$. It is reflexive, transitive and symmetric.

\section{Definition 4.1.1.}

$$
\operatorname{Know}(i, \phi, s) \stackrel{\text { def }}{=}\left(\forall s^{\prime}\right) K_{i}\left(s^{\prime}, s\right) \rightarrow \phi\left[s^{\prime}\right]
$$

This definition shows that an agent has knowledge about $\phi$ if and only if $\phi$ holds in all the epistemic possible situations of the agent. Then we can have the definition of knowledge asymmetry.

\section{Definition 4.1.2.}

$\operatorname{Know} \operatorname{Asym}(i, j, \phi, s) \stackrel{\text { def }}{=}$

$$
\operatorname{Know}(i, \phi, s) \wedge \neg \operatorname{Know}(j, \phi, s) \wedge \operatorname{Know}(i, \neg \operatorname{Know}(j, \phi, s), s)
$$


Know Asym is a fluent in situation $s$ where agent $i$ has knowledge about $\phi$ while agent $j$ does not have and this is also known by agent $i$. It can be the other way around with $i$ and $j$. But for defining opportunism, we only limit this definition to one case. Note that $\phi$ can represent any proposition in this definition.

\subsection{Value Opposition}

From the definition of opportunism, we know that agents have different evaluations on the same state transition. For agent $i$ who performs opportunistic behavior, his value gets promoted, while the value of agent $j$ gets demoted. We argue that this is because agents always make the evaluation from their perspective, which is part of their value system. This property of state transition is named value opposition in this study. In order to extend our technical framework with value theory, we define a symbol $V$ to represent agents' value system and a binary relation $<$ over situations to represent agents' preference, where $s<_{V} s^{\prime}$ denotes " $s$ ' is preferred to $s$ based on value system $V$ ".

In situation calculus, situations can be described in terms of propositions $P$, which are structured with objects and their properties. For having preferences on situations, we argue that agents evaluate the truth value of specific propositions, which are called perspectives in this study, based on their value systems. For instance, the buyer tries to see if the cup has good quality or not in order to have a preference on the situations before and after the transaction. In order to specify agents' preference on situations, we first define a function EvalRef that represents agents' perspective for evaluation:

\section{Definition 4.2.1.}

$$
\text { EvalRef : } V \times S \times S \rightarrow P
$$

It returns a proposition that an agent refers to for specifying his preference on two situations based on his value system. It is worth noting that in real life agents' specification of preferences on situations is based on a set of propositions $2^{P}$ rather than a single proposition. For instance, both whether the cup has good quality and appearance are important to the buyer. For simplicity, here we restrict the return value to only one proposition without loss of generality.

Once having this reference function, we can specify agents' preferences on situations, where $V$ is restricted to perspective-based value:

$$
\begin{aligned}
& s<_{V} s^{\prime} \equiv \operatorname{Know}(\neg p, s) \wedge \operatorname{Know}\left(p, s^{\prime}\right) \text { where } p=\operatorname{EvalRe} f\left(V, s, s^{\prime}\right) \\
& s>_{V} s^{\prime} \equiv \operatorname{Know}(p, s) \wedge \operatorname{Know}\left(\neg p, s^{\prime}\right) \text { where } p=\operatorname{EvalRe} f\left(V, s, s^{\prime}\right)
\end{aligned}
$$

It means that an agent's value gets promoted/demoted from $s$ and $s^{\prime}$ when the truth value of the proposition $p$ that the agent refers to based on his value system $V$ changes. As for the example about selling the broken cup, the seller's value gets promoted when he knows that he has earned money from the transaction, whereas the buyer's value gets demoted when he knows that the cup is broken. Because of having different value systems, they refer to different propositions and 
thereby evaluate different propositions for specifying their preferences. Similar to knowledge asymmetry, we only limit the specification to one case for the truth value of $p$.

\section{Definition 4.2.2.}

$$
\text { ValueOppo }\left(i, j, s, s^{\prime}\right) \stackrel{\text { def }}{=} s<V_{V_{i}} s^{\prime} \wedge s>_{V_{j}} s^{\prime}
$$

We define value opposition as a property of state transition where a state transition from $s$ to $s^{\prime}$ can promote the value of agent $i$ but demote the value of agent $j$. In other words, agent $i$ has positive effects from the state transition, while agent $j$ has negative effects. Again, we only limit the definition to one case for defining opportunism.

\subsection{Intention}

Opportunistic behavior is performed by intent rather than by accident. In order to suggest this aspect in our formal model, we adopt the logic of intention to do something for being something in our framework. The definition of Intend is as below:

\section{Definition 4.3.1.}

$$
\operatorname{Intend}(i, a, \phi, s) \stackrel{\text { def }}{=}\left(\forall s^{\prime}\right) I_{i}\left(s^{\prime}, s\right) \rightarrow \operatorname{occur}\left(a, s^{\prime}\right) \wedge \phi\left[s^{\prime}, \operatorname{do}\left(a, s^{\prime}\right)\right]
$$

$I\left(s^{\prime}, s\right)$ denotes the intentional accessibility relation of an agent, meaning that what is the case in situation $s^{\prime}$ is intended to have in situation $s$. occur $\left(a, s^{\prime}\right)$ is true when action $a$ is performed in situation $s^{\prime}$, and $\phi$ is true in the state transition. An intention of agent $i$ Intend $(i, a, \phi, s)$ holds if and only if both $\operatorname{occur}\left(a, s^{\prime}\right)$ and $\phi\left[s^{\prime}, d o\left(a, s^{\prime}\right)\right]$ hold in all intentional possible situations of agent $i$. Based on this definition of intention, we have two instances for value promotion $\operatorname{pro}(j)=s^{\prime}<_{V_{j}} d o\left(a, s^{\prime}\right)$ and value demotion $d e(j)=s^{\prime}>_{V_{j}} d o\left(a, s^{\prime}\right)$ by action $a$, which will be later used for providing the final definition and proving its properties

$$
\begin{aligned}
& \text { Intend }(i, a, \operatorname{pro}(j, v), s) \stackrel{\text { def }}{=}\left(\forall s^{\prime}\right) I_{i}\left(s^{\prime}, s\right) \rightarrow \operatorname{occur}\left(a, s^{\prime}\right) \wedge s^{\prime}<_{V_{j}} \operatorname{do}\left(a, s^{\prime}\right) \\
& \operatorname{Intend}(i, a, \operatorname{de}(j, v), s) \stackrel{\text { def }}{=}\left(\forall s^{\prime}\right) I_{i}\left(s^{\prime}, s\right) \rightarrow \operatorname{occur}\left(a, s^{\prime}\right) \wedge s^{\prime}>_{V_{j}} \operatorname{do}\left(a, s^{\prime}\right)
\end{aligned}
$$

Intend $(i, a, \operatorname{pro}(j), s)$ denotes that agent $i$ intends to promote the value of agent $j$ by action a in situation $s$. Similar for $\operatorname{Intend}(i, a, d e(j), s)$. When $i=j$, agent $i$ intends to promote or demote his own value by action $a$.

\subsection{Opportunistic Behavior}

The above definitions are pivotal ingredients that we need for having the formal model of opportunism: knowledge asymmetry as the precondition, value opposition as the effect, and intention as the mental state. Besides, based on the 
informal definition we gave in Sect.2, there are two more aspects that should be suggested in the definition. Firstly, the Knowledge that the performer has while others do not have should be relevant to the state transition. Secondly, the performer is aware of value opposition for the state transition beforehand but still ignores it. Opportunism is defined as follows:

Definition 4.4.1. Let $D$ be a Situation Calculus BAT, $K$ and $I$ be the axioms for knowledge and intention representation in the Situation Calculus respectively, $V$ be the value system of agents, EvalRef be the reference function representing the object for an agent's evaluation on situations, and $<_{V}$ be a preference ordering on situations. Then $\left(D \cup K \cup I, V\right.$, EvalRe $\left.f,<_{V}\right)$ is a situation calculus BAT extended with knowledge, intention, value and preference. Within this system, we have

$$
\begin{gathered}
\operatorname{Opportunism}(i, j, a, s) \stackrel{\text { def }}{=} \operatorname{Poss}(i, j, a, s) \wedge \operatorname{Intend}(i, a, \operatorname{pro}(i), s) \wedge \phi \\
\text { where } \operatorname{Poss}(i, j, a, s) \equiv \operatorname{KnowAsym}(i, j, \phi, s) \\
\phi=\operatorname{ValueOppo}(i, j, s, \operatorname{do}(a, s)) .
\end{gathered}
$$

This formula defines a predicate Opportunism where action $a$ is performed by agent $i$ to agent $j$ in the situation $s$. In this concise formula, the precondition of action $a$ is knowledge asymmetry about the state transition from $s$ to $d o(a, s)$, and action $a$ is performed by intent and results in value opposition.

Another observation from the model is about the subjectivity of opportunism. We can see through the functional fluent Eval that agents always evaluate the situations and consequently the state transition from their own perspectives, which are part of their value systems. If the value systems upon which they have evaluation change to other ones, the property of value opposition may become false. Opportunism is presented as a "problem" in most research. However, the above formal model of opportunism implies that it depends on from which perspective, or more generally value systems, we evaluate the state transition. It is positive from the perspective of agent $i$, while it is negative from the perspective of agent $j$. It is not necessarily a good thing or a bad thing; it could be either. In reality and multi-agent systems, people usually take the established norms into consideration when they decide whether it should be prevented, and the result may be different from society to society and from system to system.

After having the formal model of opportunism, we show how the propositions we informally suggest in text at the beginning is captured by our formalization.

Proposition 4.4.1. Given an opportunistic behavior performed by agent $i$ to agent $j$, both agents evaluate the behavior from different perspectives.

$$
\vDash \operatorname{Opportunism}(i, j, a, s) \rightarrow \operatorname{EvalRe} f\left(V_{i}, s, \operatorname{do}(a, s)\right) \neq \operatorname{EvalRe} f\left(V_{j}, s, \operatorname{do}(a, s)\right)
$$

Proof. If Opportunism $(i, j, a, s)$ holds, the property $\operatorname{ValueOppo}(i, j, s, d o(a, s))$ also holds. Following the definition of value opposition, we have

$$
s<_{V_{i}} d o(a, s) \wedge s>_{V_{j}} d o(a, s) .
$$


The specification of $s<_{V_{i}} d o(a, s)$ is

$$
\operatorname{Know}(i, \neg p, s) \wedge \operatorname{Know}(i, p, \operatorname{do}(a, s)) \text { where } p=\operatorname{EvalRe} f\left(V_{i}, s, d o(a, s)\right)
$$

The specification of $s>_{V_{j}} d o(a, s)$

$$
\operatorname{Know}(j, q, s) \wedge \operatorname{Know}(j, \neg q, \operatorname{do}(a, s)) \text { where } q=\operatorname{EvalRef}\left(V_{j}, s, \operatorname{do}(a, s)\right)
$$

Sentence (1) and (2) hold together. Since knowledge is consistent (i.e., the knowledge about something and the knowledge of its negation cannot exist at the same time), we have $p \neq q$, that is

$$
\operatorname{EvalRe} f\left(V_{i}, s, \operatorname{do}(a, s)\right) \neq \operatorname{EvalRef}\left(V_{j}, s, \operatorname{do}(a, s)\right) .
$$

Proposition 4.4.2. Given an opportunistic behavior performed by agent $i$ to agent $j$, agent $i$ knows the performing of this behavior demotes agent $j$ 's value, but needs not intend to get this result, which is characterized by:

$$
\begin{gathered}
\vDash \operatorname{Opportunism}(i, j, a, s) \rightarrow \operatorname{Know}\left(i, s>_{V_{j}} \operatorname{do}(a, s), s\right) \\
\not \models \operatorname{Opportunism}(i, j, a, s) \rightarrow \operatorname{Intend}(i, a, \operatorname{de}(j, v), s)
\end{gathered}
$$

Proof. The first one is already in the definition of opportunism, and we are going to prove the second one. Since the second one means that the implication does not hold in the model, what we need to do is to find a model where $\operatorname{Opportunism}(i, j, a, s)$ is true whereas $\operatorname{Intend}(i, a, d e(j), s)$ is false. The model is given as follows.

Free riding is one of the classic models about opportunism, and it occurs when someone benefits from resources, goods, or services but does not pay for them, which results in either an under-provision of those goods or services, or in an overuse or degradation of a common property resource [25]. Suppose agent $i$ is a free rider, then its behavior free riding satisfies the definition of opportunism. We have

$$
\begin{gathered}
\text { Poss }(i, \text { others, freeride, } s) \wedge \text { Intend }(i, \text { freeride, } \operatorname{pro}(i), s) \wedge \phi \\
\text { where Poss }(i, \text { others, freeride }, s) \equiv \operatorname{KnowAsym}(i, \text { others }, \phi, s) \\
\phi=\text { ValueOppo }(i, \text { others }, s, \text { do }(\text { freeride }, s)) .
\end{gathered}
$$

Then we have the two sentences below,

$$
\begin{gathered}
\left(\forall s^{\prime}\right) K_{i}\left(s^{\prime}, s\right) \rightarrow s^{\prime}>_{V_{\text {other }}} \text { do }\left(\text { freeride }, s^{\prime}\right) \\
\left(\forall s^{\prime}\right) I_{i}\left(s^{\prime}, s\right) \rightarrow \text { occur }\left(\text { freeride, } s^{\prime}\right) \wedge s^{\prime}<_{V_{i}} \text { do }\left(\text { freeride, } s^{\prime}\right)
\end{gathered}
$$

which mean that agent $i$ knows his behavior will demote the value of others, and it is his intention to promote his value by free riding.

However, the following sentence, which means that it is agent $i$ 's intention to demote the value of others, does not hold in our model,

$$
\left(\forall s^{\prime}\right) I_{i}\left(s^{\prime}, s\right) \rightarrow \operatorname{occur}\left(\text { freeride, } s^{\prime}\right) \wedge s^{\prime}>_{V_{\text {others }}} \text { do }\left(\text { freeride, } s^{\prime}\right)
$$


It is firstly because, in our formalization, we define K-relation and I-relation as two distinct types of accessibility relations such that something holds in the possible situations of knowledge does not mean that it holds in the possible situations of intention as well. Secondly, at the empirical level, agent i does not intend to reduce others' share of public goods. Therefore, Intend $(i$, freeride, de(others $), s)$ does not hold in our model.

\subsection{Opportunistic Behavior for Multiple Actions}

In the previous section, we only consider one single action as opportunistic behavior. But in real life it is common that opportunistic behavior consists of multiple actions. For instance, unlike the simple selling example at the beginning of this paper, commerce transactions between businesses usually have a couple of actions, each of which ends up with a status. In this context, the sequence of actions is opportunistic behavior instead of any single action within. Of course, a sequence of actions can be seen as one action if we only look at the precondition of the first action and the effect of the last action, but we may also investigate what properties we can derive from opportunistic behavior for multiple actions.

In situation calculus, a binary function $d o(a, s)$ is used to denote the situation resulting from performing action $a$ in situation $s$, so for a finite sequence of actions $\left[a_{1}, \ldots, a_{n}\right]$, the situation resulting from performing the sequence of actions in situation $s$ is denoted as $d o\left(a_{n}, d o\left(a_{n-1}, \ldots, d o\left(a_{1}, s\right)\right)\right)$. Therefore, based on Definition 4.1.1, the formal model of opportunistic behavior for multiple actions is given as below:

Definition 4.5.1. Let $D$ be a Situation Calculus BAT, $K$ and $I$ be the axioms for knowledge and intention representation in the Situation Calculus respectively, $V$ be the value system of agents, EvalRef be the reference function representing the object for an agent's evaluation on situations, and $<_{V}$ be a preference ordering on situations. Then $\left(D \cup K \cup I, V\right.$, EvalRe $\left.f,<_{V}\right)$ is a situation calculus BAT extended with knowledge, intention, value and preference. Within this system, we have

$$
\begin{aligned}
& \operatorname{Opportunism}\left(i, j,\left[a_{1}, \ldots, a_{n}\right], s_{1}\right) \stackrel{\text { def }}{=} \\
& \bigwedge_{1 \leq k \leq n} \operatorname{Poss}\left(i, j, a_{k}, s_{k}\right) \wedge \operatorname{Intend}\left(i, a_{k}, \operatorname{pro}(i), s_{k}\right) \wedge \phi \\
& \text { where } \operatorname{Poss}\left(i, j, a_{k}, s_{k}\right) \equiv \operatorname{KnowAsym}\left(i, j, \phi, s_{k}\right) \\
& \phi=V \text { ValueOppo }\left(i, j, s_{1}, \operatorname{do}\left(a_{n}, \operatorname{do}\left(a_{n-1}, \ldots, d o\left(a_{1}, s_{1}\right)\right)\right)\right) \\
& s_{k}=\operatorname{do}\left(a_{k-1}, \ldots, d o\left(a_{1}, s_{1}\right)\right)(1<k \leq n)
\end{aligned}
$$

Because each action in the sequence must be possible to be performed and it is the property of intention to be persistent [27], knowledge asymmetry and intention is true in $s_{k}$ for $1 \leq k \leq n$. Value opposition is the property of the state transition by the sequence of actions. A finite sequence of actions $\left[a_{1}, \ldots, a_{n}\right]$, which is performed by agent $i$ to agent $j$ in situation $s_{1}$, is opportunistic behavior 
if and only if each action is possible with the intention to promote agent $i$ 's value and the whole sequence results in value opposition for agent $i$ and $j$. This definition captures some interesting properties, which cannot be derived from Definition 4.4.1.

Proposition 4.5.1. Given a finite sequence of actions $\left[a_{1}, \ldots, a_{n}\right]$ as opportunistic behavior, we can prove that

$$
\begin{aligned}
& \vDash \operatorname{Opportunism}\left(i, j,\left[a_{1}, \ldots, a_{n}\right], s_{1}\right) \rightarrow \\
& \qquad \operatorname{KnowAsym}\left(i, j, \phi, s_{k}\right) \equiv \operatorname{KnowAsym}\left(i, j, \phi, d o\left(a_{k}, s_{k}\right)\right)(1<k<n)
\end{aligned}
$$

Proof. Each action in the sequence is possible to be performed and also

$$
\begin{gathered}
\operatorname{Poss}\left(i, j, a_{k}, s_{k}\right) \equiv \operatorname{KnowAsym}\left(i, j, \phi, s_{k}\right)(1 \leq k \leq n) \\
s_{k}=\operatorname{do}\left(a_{k-1}, \ldots, d o\left(a_{1}, s_{1}\right)\right)(1<k \leq n)
\end{gathered}
$$

Combining these two formulas, we can easily get

$$
\operatorname{KnowAsym}\left(i, j, \phi, s_{k}\right) \equiv \operatorname{KnowAsym}\left(i, j, \phi, d o\left(a_{k}, s_{k}\right)\right)(1 \leq k<n) .
$$

This proposition shows that, when opportunistic behavior consists of a sequence of actions, property knowledge asymmetry is preserved throughout the whole sequence.

Proposition 4.5.2. Given a finite sequence of actions $\left[a_{1}, \ldots, a_{n}\right]$ as opportunistic behavior, we can prove action $a_{i}$ needs not be opportunistic, which is characterized by

$$
\begin{aligned}
& \not \models \operatorname{Opportunism}\left(i, j,\left[a_{1}, \ldots, a_{n}\right], s_{1}\right)(n>1) \rightarrow \\
& \text { Opportunism }\left(i, j, a_{k}, s_{k}\right)(1 \leq k \leq n)
\end{aligned}
$$

Proof. In order to prove this proposition, we are going to find a counterexample of opportunistic behavior which satisfies $n>1$ but each action does not satisfy all the properties of opportunism.

Freeriding is still a nice model to prove this property. Since freeriding is one form of opportunistic behavior, Opportunism( $i$, others, freeride, $\left.s_{1}\right)$ is true in our model. Now we are going to split it into a sequence of actions $\left[a_{1}, \ldots, a_{n}\right]$ and suppose a free rider exist in a society with a large population and benefits from the public goods without paying. Since the amount that the free rider is supposed to pay is shared by a large population, other agents do not notice (or even not care about) the small change thus not getting their value demoted for little amount of freeriding. That is, for action $a_{k}$,

$$
\text { Know }\left(\text { others, } q, \operatorname{do}\left(a_{k}, s_{k}\right)\right) \rightarrow \neg\left(s_{k}>_{V_{j}} \operatorname{do}\left(a_{k}, s_{k}\right)\right)
$$

holds, where $q=\operatorname{EvalRe} f\left(V_{\text {others }}, s, d o\left(a_{k}, s_{k}\right)\right)$. Therefore, it is not true that

$$
\text { Opportunism }\left(i, j, a_{k}, s_{k}\right)(1 \leq k \leq n) \text {. }
$$


However, once the amount that the free rider is supposed to pay accumulates to be large enough for getting other agents' value demoted (the whole sequence of actions is considered) will it be regarded as opportunistic behavior. By theoretical comparison, this example is quite similar to Sorites paradox, where grains are individually removed from a heap of sands and the heap stops being a heap when the process is repeated for enough times [11]. So it is also interesting to think about when the behavior starts to be regarded as opportunistic.

In the example above, the fact associated with agents' preference is ignored for its small change. It is also possible that the information associated with an agent's preference is blocked. In this case, he cannot specify his preference on the situations and consequently cannot evaluate the actions. Only when he receives the specific information and compares his current situation with previous situations can the sequence of actions be considered as opportunistic behavior.

\subsection{Opportunistic Behavior with Social Context}

In the previous sections, we made an assumption for the sake of simplicity that there is no legal or moral evaluation being made or implied to opportunistic behavior such that it is not necessary good or bad. However, agents in MAS are residing in a social context which provides obligations, permissions and other types of norms for guiding agents' behaviors. The setting of those norms can also reflect the value system of a society. To have a formal model of opportunism with social context, we can of course replace the agent $j$ in our previous models with a society (in this way, we see the whole society as an agent) and get similar properties as in last two sections, but now we are more interested in putting opportunism in a deontic-based social context to see how it relates to social norms. Thus, in this section, we are going to put opportunistic behavior into a social context with norms and propose a formal model of opportunism from this perspective.

For defining opportunistic behavior with social context, we adopt the definition of knowledge asymmetry and intention in previous sections but redefine value opposition. Firstly, we have three normative statuses, which are similar to deontic logic.

- it is obligatory that $(\mathrm{OB})$

- it is permissible that $(\mathrm{PE})$

- it is forbidden that (FO)

Secondly, we define the above deontic notions for specifying the normative propositions $\Pi$.

Definition 4.6.1.

$$
\begin{gathered}
O B(i, a, s) \stackrel{\text { def }}{=}\left(\forall s^{\prime}\right) R_{i}\left(s^{\prime}, s\right) \rightarrow \operatorname{occur}\left(a, s^{\prime}\right) \\
P E(i, a, s) \stackrel{\text { def }}{=}\left(\exists s^{\prime}\right) R_{i}\left(s^{\prime}, s\right) \wedge \operatorname{occur}\left(a, s^{\prime}\right) \\
F O(i, a, s) \stackrel{\text { def }}{=}\left(\forall s^{\prime}\right) R_{i}\left(s^{\prime}, s\right) \rightarrow \neg \operatorname{occur}\left(a, s^{\prime}\right)
\end{gathered}
$$


where $R_{i}\left(s^{\prime}, s\right)$ denotes the deontic accessibility relation of agent $i$, meaning that what is the case in situation $s^{\prime}$ is ideal for situation $s$, and $\operatorname{occur}\left(a, s^{\prime}\right)$ is true when action $a$ is performed in situation $s^{\prime}$. R-relation is serial, which means for all situations $s$ there is at least one possible situation $s^{\prime}$ such that $R_{i}\left(s^{\prime}, s\right)$ holds. This property of R-relation ensures $O B(i, a, s) \rightarrow P E(i, a, s)$ to be hold, which is also consistent with our intuition. Each modality can be taken as a basic to define the other two modalities.

We then specify the social preference on situations, where $V$ is restricted to deontic-based social value.

$$
\begin{aligned}
& s<_{V_{A}} s^{\prime} \equiv(\exists a, i) s^{\prime}=d o(a, s) \wedge O B(i, a, s) \\
& s>_{V_{A}} s^{\prime} \equiv(\exists a, i) s^{\prime}=d o(a, s) \wedge F O(i, a, s)
\end{aligned}
$$

Here symbol $A$ represents the whole society, which is a set of agents. The first one means that the social value gets promoted if there exists an action whose performing complies with the social norm, while the second one means that the social value gets demoted if there exists an action whose performing violates the social norm.

Together with the specification of agents' preferences on situations, we have the definition of value opposition between agents and the whole society.

\section{Definition 4.6.2.}

$$
\text { ValueOppo }\left(i, A, s, s^{\prime}\right) \stackrel{\text { def }}{=} s<_{V_{i}} s^{\prime} \wedge s>_{V_{A}} s^{\prime}
$$

For the state transition from $s$ to $s^{\prime}$, the value of agent $i$ gets promoted whereas the social value gets demoted. We only limit the definition to one case excluding the other way around for defining opportunism.

Therefore, similar to Definition 4.4.1, we have the definition of opportunistic behavior with social context.

Definition 4.6.3. Let $D$ be a Situation Calculus BAT, $K$ and $I$ be the axioms for knowledge and intention representation in the Situation Calculus respectively, $V$ be the value system of agents, EvalRef be the reference function representing the object for an agent's evaluation on situations, $\Pi$ be a finite set of normative propositions, and $<_{V}$ be a preference ordering on situations. Then $\left(D \cup K \cup I, V\right.$, EvalRef $\left., \Pi,<_{V}\right)$ is a situation calculus BAT extended with knowledge, intention, value, norms and preference. Within this system, we have

$$
\begin{gathered}
\operatorname{Opportunism}(i, A, a, s) \stackrel{\text { def }}{=} \operatorname{Poss}(i, A, a, s) \operatorname{Intend}(i, a, \operatorname{pro}(i), s) \wedge \phi \\
\text { where } \operatorname{Poss}(i, A, a, s) \equiv \operatorname{KnowAsym}(i, A, \phi, s) \\
\phi=\operatorname{ValueOppo}(i, A, s, d o(a, s)) .
\end{gathered}
$$

Action $a$ performed by agent $i$ is regarded as opportunistic behavior if and only if it is performed with the asymmetric knowledge $\phi$ about the state transition from $s$ to $d o(a, s)$ and the intention of self-interest, and results in value opposition against the society $A$ where he is staying. 
The definition of opportunistic behavior with social context shows that, given the value system of a society, opportunistic behavior is considered to be bad since its performing results in demoting the social value. Further, it implies the moral dilemma concerning the conflict between desire and obligation. More precisely, an agent has the desire "to do what one wants", while the social context where the agent is residing gives the obligation "to do what one ought to do". Opportunistic agents follow their desire but ignore the obligation. Hence, it should be prohibited by laws or social norms from the perspective of the whole society.

Since we assume a social context with norms in this section, it is worth investigating the relation between deontic notions and mental states. Our formalization governs Proposition 4.6.1 regarding opportunistic agents having knowledge about the relevant norms, and Proposition 4.6.2 and Proposition 4.6.3 about the intention of opportunistic behavior not being derived from the obligation.

Proposition 4.6.1. Let action a be opportunistic behavior performed by agent $i$ within society $A$ in situation $s$, then for the social norm associated with action a $F O(i, a, s) \in \Pi$ we have

$$
\vDash \operatorname{Opportunism}(i, A, a, s) \rightarrow \operatorname{Know}(i, F O(i, a, s), s)
$$

Proof. Since Opportunism $(i, A, a, s)$ holds, by Definition 4.6.3, agent $i$ must have knowledge about the effect of performing action $a$, that is, $\operatorname{Know}(i, \phi, s)$ holds, where $\phi$ represents value opposition. By Definition 4.6.2, $\phi=s<_{V_{i}}$ $d o(a, s) \wedge s>_{V_{A}} d o(a, s)$. Therefore, $\operatorname{Know}\left(i, s>_{V_{A}} d o(a, s), s\right)$ holds. Because $V$ is restricted to deontic-based social value in our model, $s>_{V_{A}} \operatorname{do}(a, s) \equiv$ $F O(a, s)$ holds, thereby $\operatorname{Know}(i, F O(i, a, s), s)$ holds as well.

Agents have the knowledge about the relevant norms in the society and decide whether and which to comply with based on their own analysis. Typically, opportunistic agents behave in their interest, regardless of the social norms they are supposed to follow.

Moreover, intention might be derived from obligation (I ought to go to work this morning, so I intend to go to work this morning), or might just come from agents' own desire (I feel thirsty, so I intend to get some water). In a given situation, agents intend to perform opportunistic behavior, which is motivated by self-interest. In order to prove this property rigorously, we should first prove the disobedience of opportunistic behavior.

Proposition 4.6.2. Let action a be opportunistic behavior performed by agent $i$ within society $A$ in situation $s$, and $V_{i}$ be agent $i$ 's value system, we can prove

$$
\vDash \text { Opportunism }(i, A, a, s) \rightarrow\left(V_{i} \neq \text { Obedience }\right)
$$

Proof. By contradiction, we assume that $V_{i}=$ Obedience. Because agent $i$ obeys to the social norm in order to promote his value, action $a$ should not be forbidden by the society, that is, $F O(a, s)$ does not hold. Consequently, $s>_{V_{A}} d o(a, s)$ and Opportunism $(i, A, a, s)$ do not hold, either. Therefore, $V_{i}=$ Obedience is false for opportunistic behavior. 
Using Proposition 4.6.2, we are going to prove it does not hold in opportunistic behavior that the intention is derived from the obligation.

Proposition 4.6.3. Let action a be opportunistic behavior performed by agent $i$ within society $A$ in situation s, then for the social norm associated with action a $O B(i, a, s) \in \Pi$, we can prove

$$
\vDash \operatorname{Opportunism}(i, A, a, s) \rightarrow \neg(O B(i, a, s) \rightarrow \operatorname{Intend}(i, a, \operatorname{pro}(i), s))
$$

Proof. We can prove this proposition by contradiction. Suppose action $a$ is opportunistic behavior and sentence $O B(i, a, s) \rightarrow \operatorname{Intend}(i, a, \operatorname{pro}(i), s)$ holds in our model, which means the intended situations of agent $i$ are the subset of ideal situations, formalized as $\left(\forall s^{\prime}\right) I_{i}\left(s^{\prime}, s\right) \rightarrow R_{i}\left(s^{\prime}, s\right)$, where $s^{\prime}$ in $I_{i}\left(s^{\prime}, s\right)$ is restricted to the situation that satisfies $\operatorname{pro}(i)$. Therefore, agent $i$ intends to promote his own value and the social value by action $a$. Of course, when agent $i$ 's value is obedience, both agent $i$ 's value and the social value are promoted. But we have already proved in Proposition 4.6.2 that this possibility does not exist. So our assumption at the beginning is wrong. Therefore, $O B(i, a, s) \rightarrow$ Intend $(i, a, \operatorname{pro}(i), s)$ does not hold in our model.

\section{Example: Selling a Broken Cup}

Recall the example that we used to introduce opportunism at the beginning of the paper. The scenario is simple but enough to illustrate our formal model of opportunism. We label the seller and the buyer as $s$ and $b$, who can be in one of the situations: $S_{0}$ (the initial situation, before the transaction) and $d o\left(a, S_{0}\right)$ (after the transaction). The seller can either sell the cup $(a=\operatorname{sell}(x))$ or keep it. If the seller performs the action $\operatorname{sell}(x)$ in $S_{0}$, then situation will go to $\operatorname{do}\left(\operatorname{sell}(x), S_{0}\right)$.

In situation $S_{0}$, the asymmetric knowledge owned by the seller but not the buyer is not only about the broken cup, but also the state transition: once the transaction finishes, the situation will go from $S_{0}$ to $\operatorname{do}\left(\operatorname{sell}(x), S_{0}\right)$, which gets the value of the seller promoted whereas the value of the buyer demoted. That is, the precondition $\operatorname{Know} \operatorname{Asym}\left(s, b, \phi, S_{0}\right)$ holds. Now consider the value for both parties. Apparently both parties go for economic value. However, they have different and contradictory perspectives about the economic value. What the seller looks at is how much money he earns from the transaction. When the seller knows the broken cup has already been sold, his value gets promoted $\left(S_{0}<_{V_{s}} \operatorname{do}\left(\operatorname{sell}(x), S_{0}\right)\right.$ holds $)$. Conversely, what the buyer looks at is whether the cup has good quality or not. So when the buyer knows the cup is broken, his value gets demoted $\left(S_{0}>_{V_{b}} \operatorname{do}\left(\operatorname{sell}(x), S_{0}\right)\right.$ holds $)$. The above two sentences ensure ValueOppo $\left(s, b, S_{0}, d o\left(\operatorname{sell}(x), S_{0}\right)\right)$. Further, since it is the seller's intention to sell the broken cup to the buyer for promoting his value, sentence $\operatorname{Intend}\left(s, \operatorname{sell}(x), \operatorname{pro}(s), S_{0}\right)$ also holds. With the above formalization, we have the formula of opportunistic behavior for this example Opportunism $\left(s, b, \operatorname{sell}(x), S_{0}\right)$. 
From the above illustration, we can think of two situations that are worth considering. Firstly, if the buyer buys the cup only for decoration without using it, he will never know the cup is broken or even not care about it. In other words, the perspective of the seller getting the cup sold and the perspective of the buyer that the cup is good for decoration are not contradictory. That is, the buyer's perspective is revised to EvalRef $\left(V_{b}, S_{0}, \operatorname{do}\left(\operatorname{sell}(x), S_{0}\right)\right)=$ appearance. Because the cup has good appearance, sentence $S_{0}>_{V_{b}} d o\left(\operatorname{sell}(x), S_{0}\right)$ does not hold. In this case, the seller's behavior may not be opportunistic from the perspective of the buyer, if the social norms are not taken into account. The subjectivity of opportunism is reflected by the different judgement on the same action. Secondly, if there is nothing the seller can do except sell the broken cup when being in state $S_{0}$, it will be regarded as opportunistic behavior with the nature of selfdefense based on our first formal definition. It is because there is no moral or legal evaluation in this definition thus no matter whether the behavior is good or bad. In this sense, we can assume social context and analyze it with Definition 4.6.3. Suppose self-defense behavior is allowed by the society $\left(P E\left(i, a, S_{0}\right)\right)$. Then $S_{0}>_{V_{A}} d o\left(\operatorname{sell}(x), S_{0}\right)$ does not hold. Therefore, it is not opportunistic behavior from the perspective of the society. In our example, however, the options available to the buyer in state $S_{0}$ are $\{$ sell, keep $\}$, which means selling the broken cup is not the only action that he can perform.

Further, with the help of our model, we can gain practical insights into constraint mechanism of opportunism. In our case, one important reason why the seller's behavior is seen as opportunistic is that the seller and the buyer evaluate the state transition from two contradictory perspectives based on their value systems. In other words, even though they both go for economic value, they look at different things for evaluation. When applying this approach in collaborative relationship, it is much easier to understand how the relationship ends in defection. Therefore, one deterrence mechanism for partner opportunism is to avoid having contrasted value systems in the relationship. As for the precondition of opportunism, even though it is difficult to prevent knowledge asymmetry in business transactions, we still need to think about how much information we can provide to our partners and how they are going to use the information.

\section{Discussion}

As it is the first step of our work, we try to propose a simple but elegant model of opportunism for different context settings by making restricted assumptions. But it also means that the model might not manage to capture every possible scenarios. For instance, in Sect. 4.2 we only talk about the interaction between two agents and investigate the evaluation on the state transition from the perspectives of the two agents who are involved in the transaction. But actually such evaluation can also be done by others. Assume that a person sees the transaction and his value system is incompatible with agent $i$ 's. He may get angry with the seller even though he is not involved. In this sense, the behavior that is performed by agent $i$ is considered to be opportunistic from the perspective 
of the third agent. Further, our models only consider intentional actions. However, opportunistic behavior can also be about intentional inaction, which should really have been taken for obligation or responsibility, such as hiding important information. In this case, the social value gets demoted for agent $i$ 's not performing an obligatory action instead of performing a forbidden action. Of course, our models can capture this scenario in a way that deliberate inaction can be seen as an action. Interesting insights can be gained from further study on this part.

We also propose that the asymmetric knowledge obtained by opportunistic agents is value opposition about the state transition, which not the same as our intuition. The reason can be shown by the example in Sect. 5. Intuitively the asymmetric knowledge that the seller has is about the broken cup. Now we assume that both the seller and the buyer know the cup is broken and the seller sells it with a high price. Once the buyer knows that the broken cup is not worth that price, his value will get demoted. From that we can see it does not matter whether the fact about the broken cup is only known by one party beforehand, but whether value opposition about the transaction is only known by one party beforehand. In other words, the asymmetric knowledge is not about the objective fact, but about agents' evaluation on the state transition.

\section{Related Work}

Opportunism is not a new topic in social science. Since it was released by economist Williamson, scholars have studied the typical social behavior of economic players from various perspectives i.e. transaction cost economics [13], resource-based view [14], game theory [4], agency theory [15] and strategic management [16]. Even though they are indeed all worthwhile, it is difficult to directly apply their conclusions to MAS for improving the system's behavior because most of them are informal, which makes reasoning about this behavior in MAS impossible, and also not commonly accepted even in their own area.

In the field of artificial intelligence, there is a tradition to devise intelligent artifacts and construct intelligent system using symbolic representation of all factors involved [17]. Especially for mathematical logic, it is a greatly important approach to this field due to its highly abstract representation and reasoning about social reality. Therefore, a lot of work on logic formalism has been designed for representing and reasoning about dynamical domains such as situation calculus [18], event calculus [19] and fluent calculus [20]. We chose to use situation calculus as our basic framework because it has been well developed and extended with knowledge [9], belief [8] and other model semantics. In [9], an epistemic fluent $\operatorname{Know}(P, s)$ is proposed by adapting the standard possibleworld model of knowledge to situation calculus. We use this approach to define knowledge asymmetry where agents possess different amount of knowledge.

We integrated the notion of value into situation calculus to represent agents' preference on situations. However, in logical formalization, people usually use goals rather than value (e.g. $[12,21])$ for the same purpose. Only some works in the area of argumentation reason about agents' preferences and decision making 
by value (e.g. $[22,23])$. Even though both goals and values can be used to reason about agents' preferences about situations, they have different features. Goals are concrete and should be specified with time, place and objects. For example, to earn 1000 euros next month is a goal. If one agent's goal is achieved in one situation, then he has high evaluation on that situation. Value is described by Schwartz as trans-situational [24], which means that value is relatively stable and not limited to be applied in a specific situation. For instance, if honesty is a value of somebody, he will be honest for a long period of time. Since state transition results from the performing of actions, we can evaluate actions by whether our value is promoted or demoted in the state transition, as what we do in this study. For representing agents' evaluation on situations, Keeney and Raiffa proposed Multi-Attribute Utility Theory (MAUT) in which situations are described in terms of a set of attributes and the utilities of the situations are calculated by the sum of the scores on each attribute based on agents' value system [26]. In this study, we use a similar approach in which situations are represented through propositions and agents focus on a specific proposition based on their value systems to evaluate a state transition. Apparently, different agents may focus on different propositions thus having different evaluations on the same state transition.

\section{Conclusions}

Agents situated with information asymmetry might perform opportunistic behavior to others in their interest. Numerous works about such a social behavior have been seen in social science due to its negative effect on the relationship. However, most conclusions are based on a given form of opportunism, making it hard to build a fundamental theory that can be applied in any contexts. This study took the initiative to propose a formal model of opportunism based on the extended informal definition from Williamson. The modeling work was done based on situation calculus integrating the notion of value. We first have a preliminary model that only considers a single action between two agents, and then extend it for multiple actions with social context. Each model captures interesting properties that useful for our future research.

It is important to keep in mind that our aim is not to indicate where opportunistic behavior comes from through the model we propose, as before coming to this part we should have a thorough understanding of the nature of opportunism. Therefore, the main strength of this study is defining such a behavior from our specific perspective in a formal way, so as to represent the elements in the definition and how they relate to each other, towards building a formal system of opportunism. Only when we have a formal system can we perform further investigation on its emergence and constraint mechanism.

Further study can be carried out on the state transition within our formal system. For having a formal definition of opportunism, we defined a set of fluents that characterize the situations before and after the action is performed. However, we still have no idea how the truth value of each fluent is changed in 
the successor situation. For instance, fluent knowledge asymmetry is true in the initial situation for being a precondition of opportunism, but with our formalization we cannot prove its truth value after the action is performed. This problem can be solved by having successor state axioms for each fluent we define, which is important to representing and reasoning about the dynamics of our formal system. Of course, it is not necessary to do that for just having a formal definition. Another avenue would be to investigate how opportunism emerges based on the definition of opportunism. As we mentioned in our example, agents are not able to perform opportunistic behavior if the precondition knowledge asymmetry fails. However, it is common that agents stay in different positions with a different amount of information, so knowledge asymmetry is unavoidable. Therefore, we need to think about how much information and what kinds of information we can share with our partners. Moreover, agents' having different perspectives on the same value is the reason to value opposition of a state transition. So it is natural to think about how agents evaluate a situation from their perspectives and how the perspectives relate to their value systems for the study of opportunism emergence. Considerable insights can be achieved from the investigation about the compatibility of different value systems and the co-evolution of agents' value system with social context or environmental changes.

Acknowledgments. The research is supported by China Scholarship Council. We would like to thank anonymous reviewers, Marlo Souza, Hein Duijf and other colleagues for their helpful comments.

\section{References}

1. Williamson, O.E.: Markets and Hierarchies, Analysis and Antitrust Implications: A Study in the Economics of Internal Organization. Free Press, New York (1975)

2. Wooldridge, M.: An Introduction to Multiagent Systems. Wiley, Chichester (2009)

3. Das, T.K., Rahman, N.: Determinants of partner opportunism in strategic alliances: a conceptual framework. J. Bus. Psychol. 25(1), 55-74 (2010)

4. Cabon-Dhersin, M.-L., Ramani, S.V.: Opportunism, trust and coopera-tion a game theoretic approach with heterogeneous agents. Rationality Soc. 19(2), 203-228 (2007)

5. Chen, C.C., Peng, M.W., Saparito, P.A.: Individualism, collectivism, and opportunism: a cultural perspective on transaction cost economics. J. Manag. 28(4), 567-583 (2002)

6. McCarthy, J.: Situations, actions, and causal laws. In: Minsky, M. (ed.) Semantic Information Processing, pp. 410-417. MIT Press, Cambridge (1968)

7. Reiter, R.: Knowledge in Action: Logical Foundations for Specifying and Implementing Dynamical Systems, vol. 16. MIT press, Cambridge (2001)

8. Shapiro, S., Pagnucco, M., Lesprance, Y., Levesque, H.J.: Iterated belief change in the situation calculus. Artif. Intell. 175(1), 165-192 (2011)

9. Scherl, R.B., Levesque, H.J.: Knowledge, action, and the frame problem. Artif. Intell. 144(1), 1-39 (2003)

10. Demolombe, R., Parra, P.P.: Integrating state constraints and obligations in situation calculus. Inteligencia Artificial, Revista Iberoamericana de Inteligencia Artificial 13(41), 54-63 (2009) 
11. Hyde, D.: Sorites paradox. In: Zalta, E.N.(ed.).: The Stanford Encyclopedia of Philosophy (Winter 2014 Edition). http://plato.stanford.edu/archives/win2014/ entries/sorites-paradox/

12. Cohen, P.R., Levesque, H.J.: Intention is choice with commitment. Artif. Intell. 42(2), 213-261 (1990)

13. Williamson, O.E.: The Economic Institutions of Capitalism. Free Press, New York (1985)

14. Conner, K.R., Prahalad, C.K.: A resource-based theory of the firm: knowledge versus opportunism. Organ. Sci. 7(5), 477-501 (1996)

15. Jiraporn, P., et al.: Is earnings management opportunistic or beneficial? an agency theory perspective. Int. Rev. Financ. Anal. 17(3), 622-634 (2008)

16. Yaqub, M.Z.: Antecedents, consequences and control of opportunistic behavior in strategic networks. J. Bus. Econ. Res. (JBER) 7(2), 15-31 (2009)

17. Segerberg, K., Meyer, J.-J., Kracht, M.: The logic of action. In: Zalta, E.N.(ed.).: The Stan-ford Encyclopedia of Philosophy (Winter 2013 Edition). http://plato. stanford.edu/archives/win2013/entries/logic-action/

18. McCarthy, J., Hayes, P.: Some philosophical problems from the standpoint of artificial intelligence. Stanford University, USA (1968)

19. Kowalski, R., Sergot, M.: A logic-based calculus of events. In: Schmidt, J.W., Thanos, C. (eds.) Foundations of Knowledge Base Management: Contributions from Logic, Databases, and Artificial Intelligence Applications. Topics in Information Systems, pp. 23-55. Springer, Heidelberg (1989)

20. Thielscher, M.: Introduction to the fluent calculus. Electron. Trans. Artif. Intell. 2(3-4), 179-192 (1998). http://www.etaij.org

21. Rao, A.S., Georgeff, M.P.: Modeling rational agents within a BDI-architecture. In: KR 1991, pp. 473-484 (1991)

22. Bench-Capon, T., Atkinson, K., McBurney, P.: Using argumentation to model agent decision making in economic experiments. Auton. Agent. Multi Agent Syst. 25(1), 183-208 (2012)

23. van der Weide, T.L.: Arguing to motivate decisions. SIKS Dissertation Series 2011 (2011)

24. Schwartz, S.H.: Universals in the content and structure of values: theoretical advances and empirical tests in 20 countries. In: Advances in experimental social psychology, vol. 25, pp. 1-65 (1992)

25. Baumol, W.J.: Welfare Economics and the Theory of the State. Harvard University Press, Cambridge (1967)

26. Keeney, R.L., Raiffa, H.: Decisions with Multiple Objectives: Preferences and Value Trade-offs. Cambridge University Press, Cambridge (1993)

27. Bratman, M.: Intention, Plans, and Practical Reason. Harvard University Press, Cambridge (1987) 